\title{
The Phosphate Transporter Gene OsPht1;4 Is Involved in Phosphate Homeostasis in Rice
}

\author{
Ying Ye, Jing Yuan, Xiaojian Chang, Meng Yang, Lejing Zhang, Kai Lu, Xingming Lian* \\ National Key Laboratory of Crop Genetic Improvement and National Center of Plant Gene Research \\ (Wuhan), Huazhong Agricultural University, Wuhan, 430070, P.R. China \\ *xmlian@mail.hzau.edu.cn
}

\section{Abstract}

A total of 13 phosphate transporters in rice (Oryza sative) have been identified as belonging to the Pht1 family, which mediates inorganic phosphate (Pi) uptake and transport. We report the biological property and physiological role of OsPht1;4 (OsPT4). Overexpressing OsPT4 resulted in significant higher $\mathrm{Pi}$ accumulation in roots, straw and brown rice, and suppression of OsPT4 caused decreased Pi concentration in straw and brown rice. Expression of

\section{Open ACCEss}

Citation: Ye Y, Yuan J, Chang X, Yang M, Zhang L, Lu K, et al. (2015) The Phosphate Transporter Gene OsPht1;4 Is Involved in Phosphate Homeostasis in Rice. PLOS ONE 10(5): e0126186. doi:10.1371/ journal.pone. 0126186

Academic Editor: Hatem Rouached, INRA, FRANCE

Received: December 16, 2014

Accepted: March 30, 2015

Published: May 13, 2015

Copyright: $\odot 2015$ Ye et al. This is an open access article distributed under the terms of the Creative Commons Attribution License, which permits unrestricted use, distribution, and reproduction in any medium, provided the original author and source are credited.

Data Availability Statement: All relevant data are within the paper and its Supporting Information files.

Funding: This work was supported by grants from the National Basic Research Program of China (2011CB100304), the National High Technology Research and Development Program of China (2012AA10A303), the National Natural Science Foundation of China (31172017), the transgenic project (2014ZX08001005) and the Gates Foundation. The funders had no role in study design, data collection and analysis, decision to publish, or preparation of the manuscript. the $\beta$-glucuronidase reporter gene driven by the OsPT4 promoter showed that OsPT4 is expressed in roots, leaves, ligules, stamens, and caryopses under sufficient $\mathrm{Pi}$ conditions, consistent with the expression profile showing that OsPT4 has high expression in roots and flag leaves. The transcript level of OsPT4 increased significantly both in shoots and roots with a long time Pi starvation. OsPT4 encoded a plasma membrane-localized protein and was able to complement the function of the Pi transporter gene PHO84 in yeast. We concluded that OsPT4 is a functional Pi-influx transporter involved in $\mathrm{Pi}$ absorption in rice that might play a role in Pi translocation. This study will enrich our understanding about the physiological function of rice Pht1 family genes.

\section{Introduction}

Phosphorous is a major macronutrient for plant growth and development [1]. Inorganic phosphorus $(\mathrm{Pi})$ ensures the maintenance of metabolism and the processes of cell formation and signal transduction [2-4]. To resolve the conflict between the root peripheral environment with low $\mathrm{Pi}$ concentrations $(<10 \mu \mathrm{m})$ and high Pi concentrations (5-20 mM) in plant tissues [5], plants have formed an effective system to absorb and distribute $\mathrm{Pi}$ as much as possible. Two kinds of Pi transporters (PTs) have evolved to mediate Pi absorption and transport in rice: one is the high-affinity Pi transporter (with $\mathrm{Km}$ values in the micromolar concentration range) and the other is the lowaffinity transporter (millinolar levels) [6-8]. After the first high-affinity Pi transporter PHO84 was found in yeast (Saccharomyces cerevisiae), many other Pi transporters, such as 19 genes in Arabidopsis and 26 genes in rice, have been identified [1,9]. Based on their difference in structure and subcellular localization, all Pi transporters from dicot and monocot plants have been grouped into four subfamilies, PHT1 to PHT4, which are mainly localized to plasma membrane, chloroplast, mitochondria, and Golgi apparatus, respectively [10]. 
Competing Interests: The authors have declared that no competing interests exist.
In Arabidopsis, five of the nine genes in the Pht 1 family, which contains AtPht1;1, AtPht1;4, AtPht1;5, AtPht1;8, and AtPht1;9, have been isolated and functionally characterized [11,12]. In general, all five transporters belong to the high-affinity Pi transport system and are significantly affected by low Pi. AtPht1;1 and AtPht 1;4 are capable of Pi transport and are localized mainly at the plasma membrane of epidermis and stele cells of Arabidopsis roots, confirming their important roles in Pi acquisition and root-to-shoot translocation [13]. AtPht1;8 and AtPht1;9 also show high expression levels in roots, suggesting their potential in root Pi uptake and translocation [14]. In contrast to those four genes, AtPht 1;5 is expressed in shoots and actively involved in the mobilization of phosphorous from the Pi source to sink organs in accordance with Pi status [15]. Aside from these transporters in Arabidopsis, many PT family genes from other species have also been characterized, such as PHO5 from Neurospora crassa [16], GvPT from Glomus versiforme [16,17], StPT1 and StPT2 from potato [18], LePT1 and LePT2 from tomato [19,20], MtPT1 and MtPT2 from Medicago [21], and HvPT1, HvPT2, and HvPT6 from barley [22-24]. All these genes have been shown to encode functional Pi transporter proteins and to be involved in Pi uptake or translocation.

So far, 13 genes have been isolated in the rice Pht 1 family and eight members have been functionally characterized [25]. Among them, OsPT1, OsPT6, OsPT9, and OsPT10 are highly expressed in roots and responsible for absorbing Pi in rice [26-28]. OsPT1 is constitutively expressed in roots, indicating that this gene plays an important role in Pi acquisition under various Pi conditions [26]. In contrast to OsPT1, the other three genes are increasingly expressed by low Pi stress and function at low Pi conditions. After being taken-up by rice roots, most Pi should be transported into shoots and then distributed into various organs. This process is complex and requires the cooperation of many Pi transporters. OsPT2 and OsPT8 are both highly expressed in stele cells of rice roots and leaves of shoots, and they play essential roles in Pi root-to-shoot translocation and Pi homeostasis in shoots [29,30]. Moreover, OsPT8 also contributes to Pi translocation from the panicle axis to the rice hull. Also, OsPT11 and OsPT13 appear to be involved in arbuscular mycorrhizal fungal symbiosis [31]. Although studies of Pht1 family genes in rice have lasted decades, there are still a few Pi transporters that need to be characterized. In this report, we investigated the function of OsPht1;4 (accession no. AF 536964 ) in rice. We found that OsPT4 is a functional Pi transporter localized mainly in the plasma membrane of exodermis cells in rice roots. Overexpressing OsPT4 induced an increase in Pi concentration at different Pi levels. We concluded that OsPT4 is involved in Pi uptake from an external solution.

\section{Material and Methods}

\section{Plant Materials and Growth Condition}

Based on the Oryza sativa ssp. Japonica cv. Nipponbare background, overexpression and knockdown lines of OsPT4 were obtained via Agrobacterium tumefaciens-mediated transformation [32].

Standard rice culture solution was used in hydroponic experiments. The dispensation of culture solution was as follows: $1.44 \mathrm{mM} \mathrm{NH}_{4} \mathrm{NO}_{3} ; 0.5 \mathrm{mM} \mathrm{K}_{2} \mathrm{SO}_{4} ; 1.0 \mathrm{mM} \mathrm{CaCl}_{2} ; 1.6 \mathrm{mM}$ $\mathrm{MgSO}_{4} ; 0.17 \mathrm{mM} \mathrm{Na}_{2} \mathrm{SiO}_{3} ; 0.3 \mathrm{mM} \mathrm{NaH}_{2} \mathrm{PO}_{4} ; 50 \mu \mathrm{M} \mathrm{Fe}-\mathrm{EDTA} ; 0.06 \mu \mathrm{M}\left(\mathrm{NH}_{4}\right)_{6} \mathrm{Mo}_{7} \mathrm{O}_{24}$; $15 \mu \mathrm{M} \mathrm{H}_{3} \mathrm{BO}_{3} ; 8 \mu \mathrm{M} \mathrm{MnCl}_{2} ; 0.12 \mu \mathrm{M} \mathrm{CuSO}_{4} ; 0.12 \mu \mathrm{M} \mathrm{ZnSO}_{4} ; 29 \mu \mathrm{M} \mathrm{FeCl}_{3}$; and $40.5 \mu \mathrm{M}$ citric acid ( $\mathrm{pH}$ 5.5) [33]. The transgenic lines were grown in solution containing different concentrations of phosphate with $0.3 \mathrm{mM} \mathrm{NaH} \mathrm{PO}_{4}$ (normal treatment), $1.5 \mathrm{mM} \mathrm{NaH}_{2} \mathrm{PO}_{4}$ (high-Pi treatment [HP]), and $0 \mathrm{mM} \mathrm{NaH}_{2} \mathrm{PO}_{4}$ (low-Pi treatment [LP]). The solution was renewed every 5 days. 


\section{Microarray Expression Profile}

To analyze the expression pattern of OsPT4, genome-wide expression data were searched in the CREP database (http://crep.ncpgr.cn/crep-cgi/home.pl) [34]. All these data were generated by hybridizing Affymetrix whole-genome arrays from RNA samples obtained from 27 tissues collected throughout the life cycle of three genotypes of cultivated rice: Minghui 63, Zhenshan 97, and their hybrid Shanyou 63.

\section{Histochemical Localization of GUS Expression}

The construct of OsPT4 promoter GUS was transformed into Nipponbare via Agrobacterium tumefaciens-mediated transformation [35]. The transgenic lines were grown in solution with or without $\mathrm{Pi}$ and the tissues and organs were incubated in $\mathrm{X}-\mathrm{Glu}$ strain buffer at $37^{\circ} \mathrm{C}$ overnight [36]. To observe the cellular expression patterns, the stained tissues were rinsed in $70 \%$ ethanol for 24 hours, embedded in agarose gel, and then sectioned. The stained tissues were photographed using DFC290 stereomicroscope with a color CCD camera and sections were visualized with a digital camera DXM1200C.

\section{Subcellular Location of OsPT4}

The coding sequence of OsPT4 (LOC_Os04g10750) from the RGAP database (http://crep. ncpgr.cn/crep-cgi/home.pl) was amplified and transformed into vector pM999-GFP. The preparation and transfection of rice protoplasts were performed as described [37]. Briefly, $0.1 \mathrm{~mL}$ of protoplast suspension (approximately $1 \times 10^{5}$ cells) was transfected with $10 \mu \mathrm{g}$ of DNA via 0.12 $\mathrm{mL} 40 \%$ PEG. After 12 to 24 hours of incubation at $22^{\circ} \mathrm{C}$, the cells were observed by a confocal laser scanning microscope (TCS SP2).

\section{RNA Extraction and Real-Time PCR}

Total RNA was extracted using TRizol regent (Invitrogen). According to the manufacturer's instructions, $3 \mu \mathrm{g}$ of total RNA was used to synthesize the first-strand cDNA in $20 \mu \mathrm{l}$ of reaction mixture using M-MLV reverse-transcriptase (Invitrogen). Real-time PCR was performed using the SYBR Premix Ex Taq ${ }^{\mathrm{TM}}$ (TaKaRa) with the gene-specific primers listed in Table 1. The real-time PCR reaction was performed on an Applied Biosystems 7500 PCR instrument. The rice Ubiquitin 5 gene was used as the internal control.

The functional complementation experiment was performed to explore the Pi transport activity of OsPT4. The OsPT4 open reading frame was first cloned into pGEM-T Easy vector and digested with $\mathrm{xbaI}$, and then correctly introduced into vector $p Y E S 2$. The resulting plasmid was transformed into yeast strain $\Delta$ pho84 (Mat a; his3 $\Delta 1$; leu2 $\Delta 0$; met15 $\Delta 0$; ura3 $\Delta 0$; YML123c:: kanMX4) and its wild-type BY4741 (Mat a; his3 $\Delta 1$; leu2 $\Delta 0$; met15 $\Delta 0$; ura3 $\Delta 0$ ) purchased from EUROSCARF (http://web.uni-frankfurt.de/fb15/mikro/euroscarf/index.html) [38]. The pYES2-OsPT4 and control cells were grown to the logarithmic phase and then subjected to yeast nitrogen base liquid medium with different Pi concentrations of $0 \mu \mathrm{M}$ and $75 \mu \mathrm{M}$, respectively. Bromcresol purple was used as the $\mathrm{pH}$ indicator in the medium.

\section{Measurement of Pi Concentration in Plants}

The measurement of inorganic Pi was performed as described previously with some modifications. Fresh samples were milled in liquid nitrogen and were not placed on ice until they thawed. Then, the milled samples were homogenized in $10 \%(\mathrm{w} / \mathrm{v})$ perchloric acid: $5 \%(\mathrm{w} / \mathrm{v})$ perchloric acid (1:9) and placed on ice for 30 minutes. After centrifugation at 10,000 $\mathrm{g}$ for 10 minutes at $4^{\circ} \mathrm{C}$, the supernatant was used for Pi measurement using the molybdenum blue method. The working 
Table 1. Primers used in this study.

\begin{tabular}{|c|c|}
\hline Primer Name & Sequence $\left(5^{`}-3^{`}\right)$ \\
\hline OE-OsPT4-F & ATGGATCCAATTGCAGCGAGATTTTGTC \\
\hline OE-OsPT4-R & TACTGCAGTGGTACACTAGCAGAACCAGAA \\
\hline GFP- OsPT4-F & TCTAGAATGGCCGGCGAGCTCAAGGTG \\
\hline GFP- OsPT4-R & TCTAGAAGCTGGCGGCGCCGGC \\
\hline pyes2- OsPT4-F & AAGCTTATTGCATATTGCAGAGTAGCTG \\
\hline pyes2- OsPT4-R & GGATCCATGATTGACCATTTGGGTTTT \\
\hline GUS- OsPT4-F & TGATTGATGAAACTGCTGCTG \\
\hline GUS- OsPT4-R & ACATATCCAGCCATGCACACT \\
\hline qRT-OsPT4-F & GCAACGTCATCGGGTTCTTCTTCA \\
\hline qRT-OsPT4-R & ACATATCCAGCCATGCACACT \\
\hline qRT-OsPHO2-F & GGCTATCGGAACTTATGG \\
\hline qRT-OsPHO2-R & AAGAAGGCAGAGGAGGTATC \\
\hline qRT-OsPHR2-F & CGCTTTGTAGATGCTGTCAATC \\
\hline qRT-OsPHR2-R & AGACCCTCATCACATCCTCATTATC \\
\hline qRT-OsSPX1-F & GACCAGCTTCTACCATCAAACG \\
\hline qRT-OsSPX1-R & AGTTCCTGCTGCTCCTCTGG \\
\hline qRT-Ubiquitin-F & AACCAGCTGAGGCCCAAGA \\
\hline qRT-Ubiquitin-R & ACGATTGATTTAACCAGTCCATGA \\
\hline
\end{tabular}

Functional Complementation Assay of OsPT4 in Yeast

doi:10.1371/journal.pone.0126186.t001

fluid is a 6:1 ratio intermixture of $0.4 \%(\mathrm{w} / \mathrm{v})$ ammonium molybdate dissolved in $0.5 \mathrm{M} \mathrm{H}_{2} \mathrm{SO}_{4}$ mixed with $10 \%$ ascorbic acid. Two milliliters of intermixture was added to $1 \mathrm{~mL}$ of sample solution, incubated in a water bath at $42^{\circ} \mathrm{C}$ for 20 minutes, and then cooled on ice. Sample absorbance was measured at $820 \mathrm{~nm}$, and Pi concentration was calculated by normalization to fresh weight values [39]. For the measurement of total Pi concentration in the rice, about $0.2 \mathrm{~g}$ dry samples were used following the method described by Chen et al [40].

\section{Results}

\section{The Expression Pattern of OsPT4 in Different Tissues of Rice}

To clarify OsPT4 gene function in detail, the expression patterns in different tissues were extracted from the CREP database; the microarray data were verified by quantitative PCR to reveal the expression profile of OsPT4. The average signal values in Fig $1 \mathrm{~A}$ were tested in 27 tissues or organs over the entire lifecycle of the rice plant in three genotypes: Minghui 63, Zhenshan 97, and Shanyou 63. Each sample was tested with two biological repeats. In general, OsPT4 exhibited the strongest expression signals in vegetative organs, such as flag leaves and roots. OsPT4 also showed a moderate expression level in calli, embryo, and radical. We also tested the transcript level of OsPT4 in different tissues of Nipponbare by Real-time PCR. Consistent with prior results, the highest expression of OsPT4 was observed in flag leaves, followed by roots (Fig 1B).

According to previous work, the expression of Pht1 family genes is largely increased under phosphate-deficient conditions [8]. To investigate whether OsPT4 is affected by low Pi treatment, we performed a time-course experiment under Pi-free and Pi-resupplied conditions. Real-time PCR showed that the transcript level of OsPT4 in roots was not significantly changed when rice plants were hydroponically cultivated under Pi-free conditions for 7 days, but the 

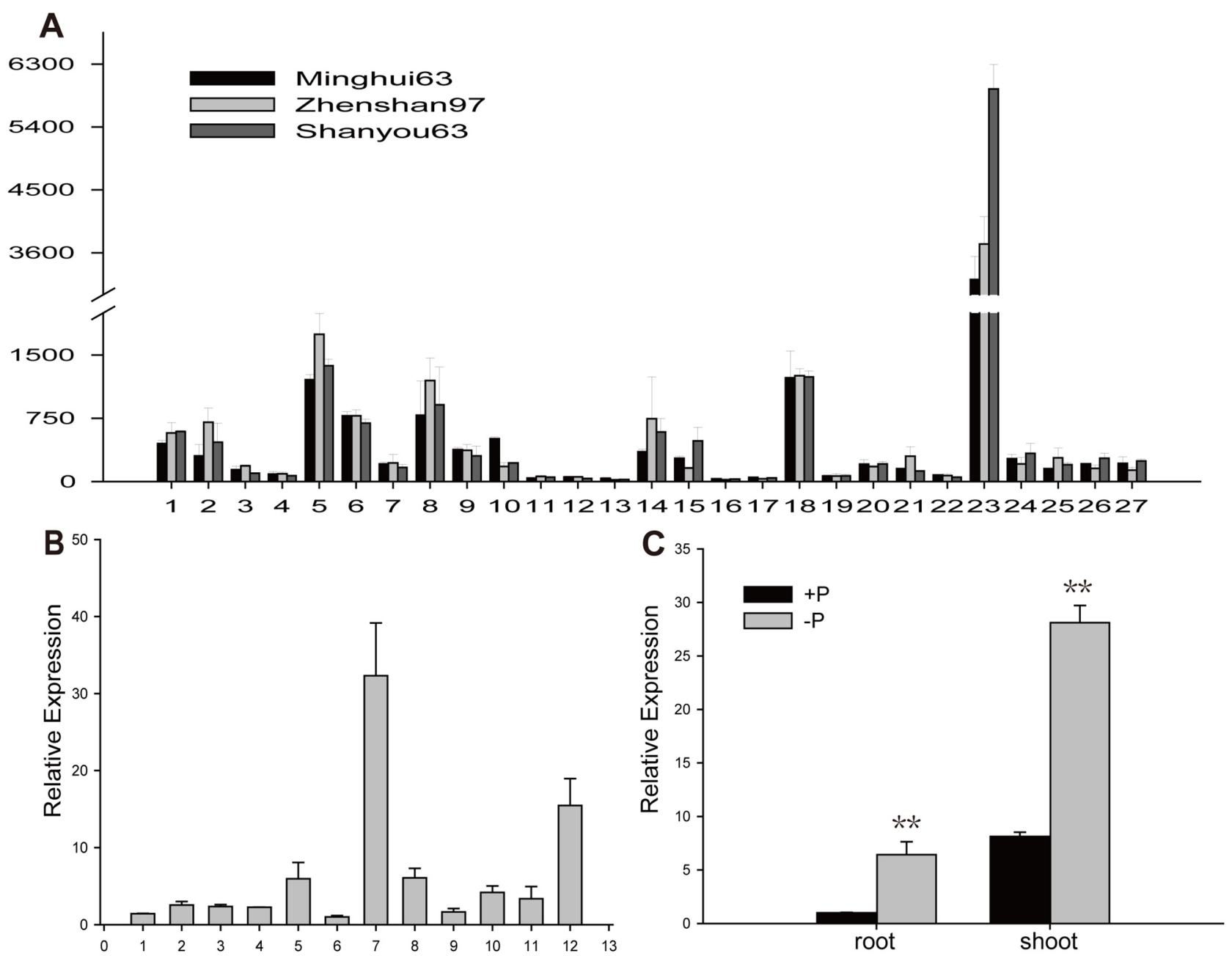

Fig 1. Expression profile of OsPT4 and expression pattern in response to Pi availability. A: The Expression profile of OsPT4 gene exhibited tissue- or organ-preferential expression patterns in 27 tissues covering the rice lifecycle based on average signal values in three rice cultivars: Minghui 63 , Zhenshan 97 and Shanyou63, respectively. For relevance, there were 27 tissues analyzing in the study: (1) calli at $15 \mathrm{~d}$ after the subculture stage; (2) resistance calli at the screening stage; (3) calli at $5 \mathrm{~d}$ after the regeneration stage; (4) seed germination after $72 \mathrm{~h}$ of the stage; (5) embryo and radical after the germination stage; (6)leaf and root at three-leaf stage; (7) shoot at the seedling with two-tillers stage; (8) root at the seedling with two-tillers stage; (9) leaf at the 1-mm young panicle stage; (10) sheath at the 1-mm young panicle stage; (11) panicle at the 1-mm young panicle stage; (12) panicle at the pistil/stamen primordial differentiation stage; (13) panicle at the pollen/mother cell formation stage; (14) leaf at the 4-5-cm young panicle stage; (15) sheath at the 4-5-cm young panicle stage; (16) panicle at the 4-5-cm young panicle stage; (17) stem at $5 \mathrm{~d}$ before the heading stage; (18) flag leaf at $5 \mathrm{~d}$ before the heading stage; (19) stem at the heading stage; (20) panicle at the heading stage; (21) hull at $1 \mathrm{~d}$ before the flowering stage; (22) stamen at $1 \mathrm{~d}$ before the flowering stage; (23) flag leaf at $14 \mathrm{~d}$ after the heading stage; (24) spikelet at $3 \mathrm{~d}$ after the pollination stage; (25) endosperm at $7 \mathrm{~d}$ after the pollination stage; (26) endosperm at $14 \mathrm{~d}$ after the pollination stage; and (27) endosperm at $21 \mathrm{~d}$ after the pollination stage. B: The relative expression of OsPT4 in different 12 tissues of Nipponbare. There were 12 tissues analyzing in the study: (1)flag leaf at 5 days before heading; (2)young panicle at 5 days before heading; (3)flag leaf at heading stage; (4)panicle at heading stage; (5)stem at heading stage; (6)panicle at one day before flowering; (7) flag leaf at 7 days after pollination; (8)culm at 7 days after pollination; (9) spikelet at 7 days after pollination; (10) culm at 14 days after pollination; (11)spikelet at 14 days after pollination; (12) root at 14 days after pollination. C: Detection of the expression alternation of OsPT4 in wild-type plants roots and shoots by real-time PCR analysis. Plants were grown to five-leaf stage in full nutrient solution, then transplanted into the solution without $\mathrm{Pi} 21$ days. Total RNA were extracted from plant tissues which were sampled at different time. Error bars indicate $\pm S D(n=3) .\left({ }^{* *} p<0.01\right)$.

doi:10.1371/journal.pone.0126186.g001

expression of OsPT4 in shoots increased with extended Pi deficiency, and quickly returned to normal levels after Pi was resupplied (S1 Fig). However, when the Pi-free conditions extend for 21 days, the transcript level of OsPT4 increased significantly both in shoots and roots (Fig 1C).

For histochenmical analysis, a construct containing a 1990-bp promoter and a GUS reporter gene was transformed into rice (cv Nipponbare) by Agrobacterium-mediated transformation. 
The transgenic plants carrying OsPT4::GUS were cultured in nutrient solution and stained for GUS activity. Strong GUS activity was detected in roots, leaves, ligules, stamens, and caryopses under sufficient Pi conditions (Fig 2), consistent with the expression profile described. In roots, OsPT4 was mainly expressed in the tip and the mature zone of roots. We performed transverse sections of these two zones and found that OsPT4 was specifically localized to the exodermis layer (Fig 2E and 2F). Since the transcript level of OsPT4 was significantly increased under Pideprived condition, the GUS activity of the transgenic plants at Pi-free condition for 21 days was detected. Consistent with the result shown by Real-time PCR, the GUS activities in roots and shoots were both enhanced under Pi-deprived condition. Moreover, GUS activity was remarkably observed in root cortex of plants cultivated under Pi-deprived conditions whereas it was not detected in the root cortex under Pi- replete conditions (S2 Fig).
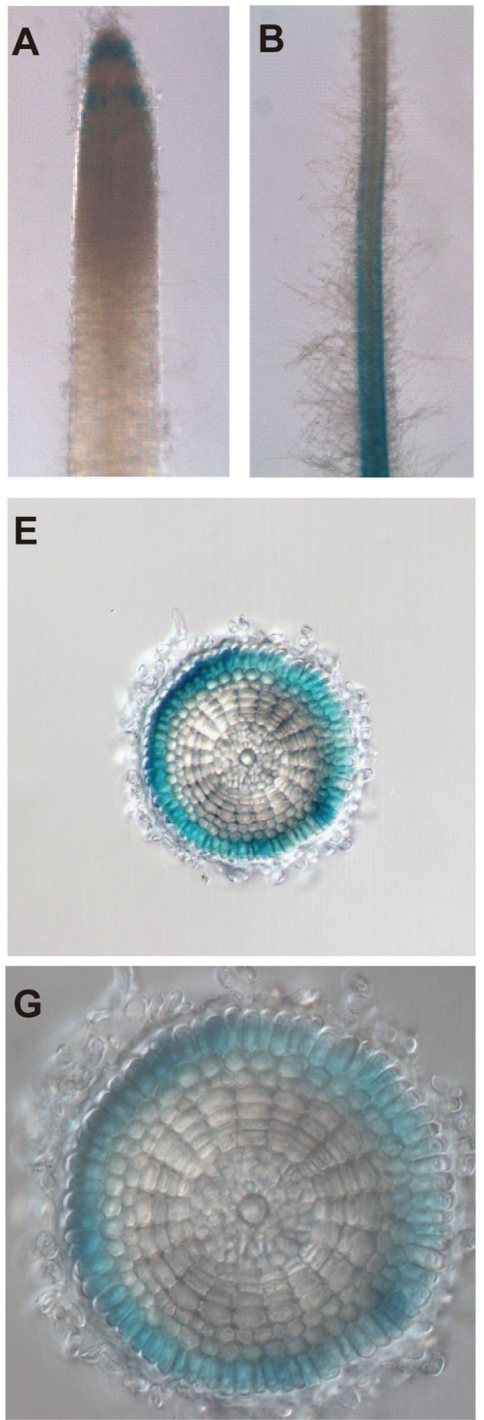
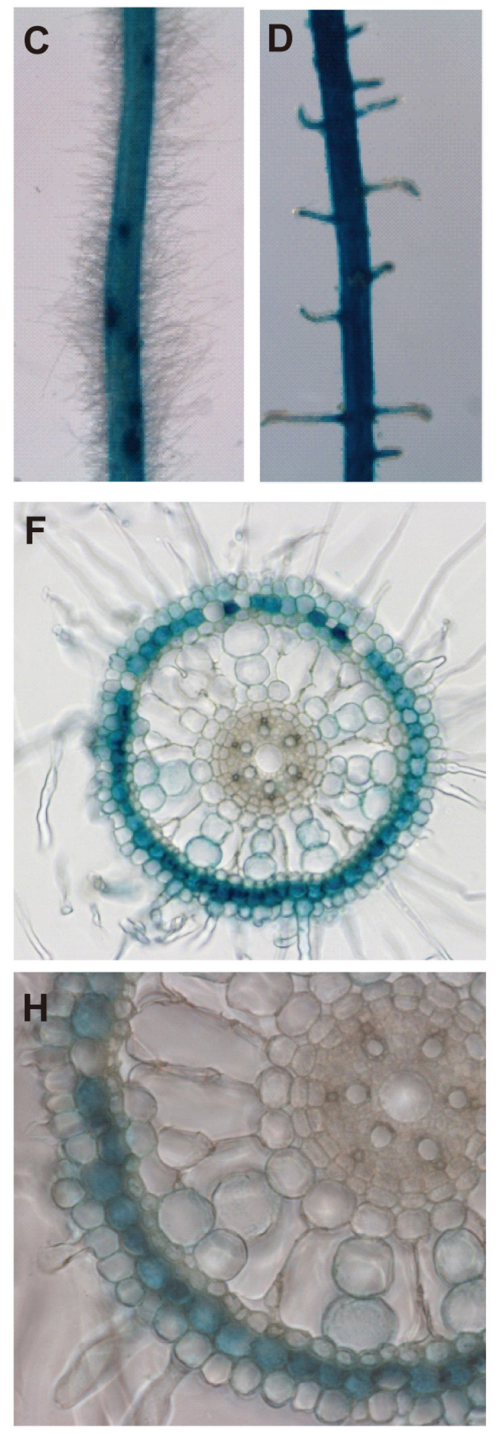

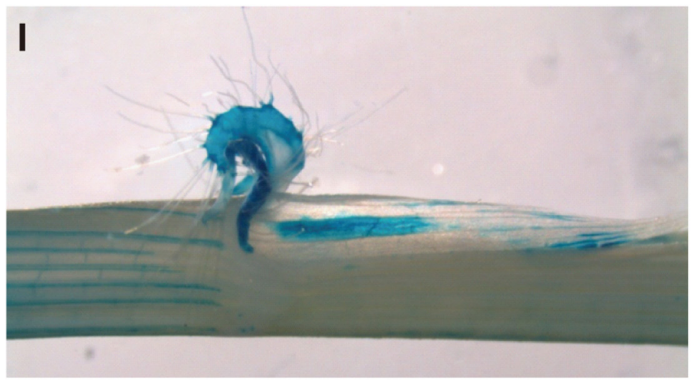

$\mathbf{J}$
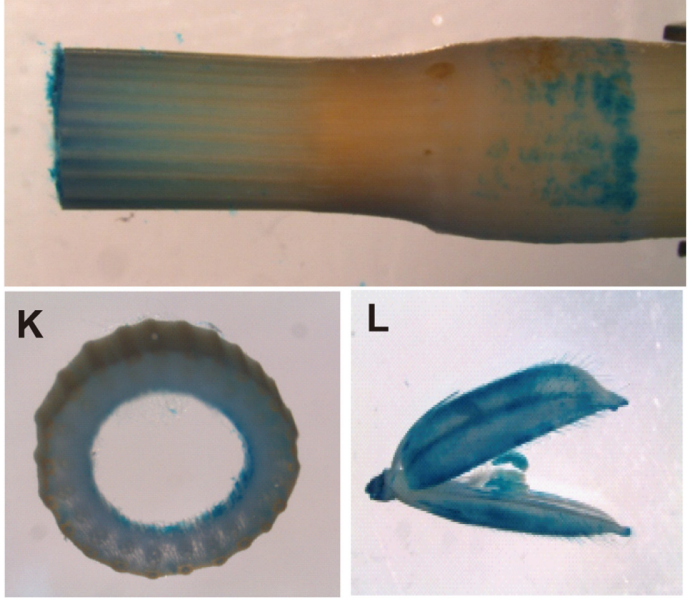

M

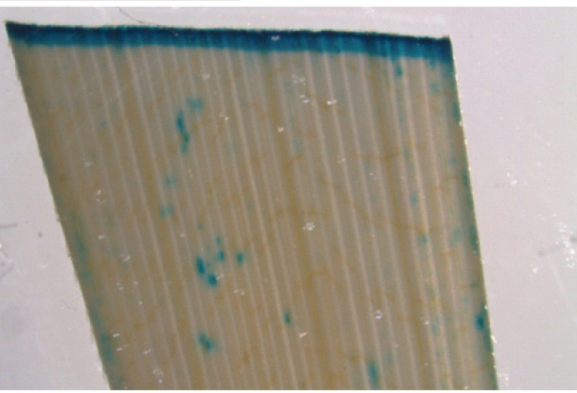

Fig 2. Tissue localization of OsPT4. GUS staining of transgenic plants harboring the OsPT4 promoter. GUS fusion was observed. Expression of OsPT4 was shown in different tissues of rice supplied with normal Pi for 21 days. A: Root tip. B: The junction between the meristematic and elongated zones. C: Branching of lateral root. D: Lateral root. E: Transverse section of root tip. F: Transverse section of root maturation zone. G and H: Enlarged images of (E) and (F). I: Ligule. J: Internodes of culm. K: Transverse section of culm. L: Caryopse and stamen. M: Leaf blade. 

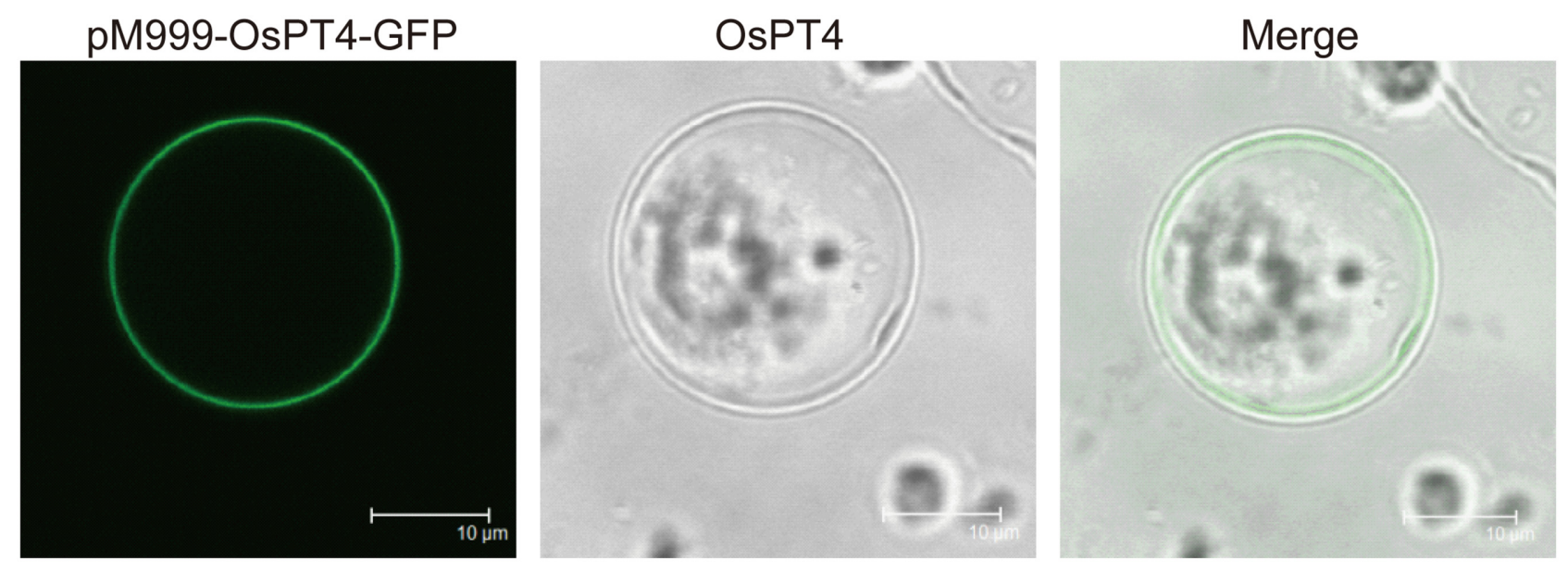

Fig 3. Subcellular localization of OsPT4 protein. Subcellular localization of OsPT4 protein was determined in rice protoplasts. The confocal image was acquired using a confocal laser scanning microscope (TCS SP2).

doi:10.1371/journal.pone.0126186.g003

\section{Subcellular Location of OsPT4}

Plant Pht1 members were predicted to be located on the plasma membrane. For confirmation of the subcellular location of OsPT4, we created an OsPT4::GFP construct at C-terminal and transfected it into rice protoplasts. As expected, the green fluorescence of OsPT4 was observed in the plasma membrane by laser scanning confocal microscopy (Fig 3).

\section{Functional Assay of OsPT4 in Yeast}

Because OsPT4 belongs to the Pht 1 family, which is always involved in Pi transport, a functional complementation analysis of the yeast mutant was performed. Yeast cell growth was wellcorrelated with acidification of the liquid growth media, as indicated by the color shift of the $\mathrm{pH}$ indicator bromocresol purple. Bromocresol purple changes its color from blue to yellow at $\mathrm{pH}$ from 6.3 to 5.8. We expressed the OsPT4 in the yeast mutant strain $\Delta p h o 84$, which is defective in high-affinity Pi transport. The mutant yeast containing OsPT4 and empty vector were grown in a yeast nitrogen base medium with various concentrations of Pi over 10 hours at $\mathrm{pH}$ 7.0 The $\mathrm{pH}$ indicator did not change from blue to yellow until the yeast cells had significant growth in culture. The colors of these three yeasts containing wild-type and $\Delta p h o 84$ with and without OsPT4 were all blue at the Pi concentration of $0 \mu \mathrm{M}$, revealing that little or no yeast cells had grown in medium without Pi. After increasing the Pi concentration of the medium to $75 \mu \mathrm{M}$, the color changed to yellow, with wild-type and $\Delta$ pho84 expressing OsPT4 rather than mutant yeast, suggesting that the expression of OsPT4 restored the yeast growth rate (Fig 4A). To further confirm this result, absorbance of these different yeasts at $600 \mathrm{~nm}$ was tested and showed that OsPT4 could partially complement the function of yeast Pi transporter PHO84 (Fig 4B).

\section{Overexpression of OsPT4 Increased Pi Accumulation in Roots}

To further examine the exact role that OsPT4 plays in rice Pi uptake and translocation, we generated overexpression lines using the CaMV $35 \mathrm{~S}$ promoter (OsPT4-Oe) and knockdown lines using RNAi (OsPT4-Ri) in Nipponbare via Agrobacterium tumefaciens-mediated transformation. Expression levels of the OsPT4 gene in the transgenic lines were tested by real-time PCR, and two OsPT4-Oe lines (Oe1 and Oe2) and two OsPT4-Ri lines (Riland Ri2) were selected for 

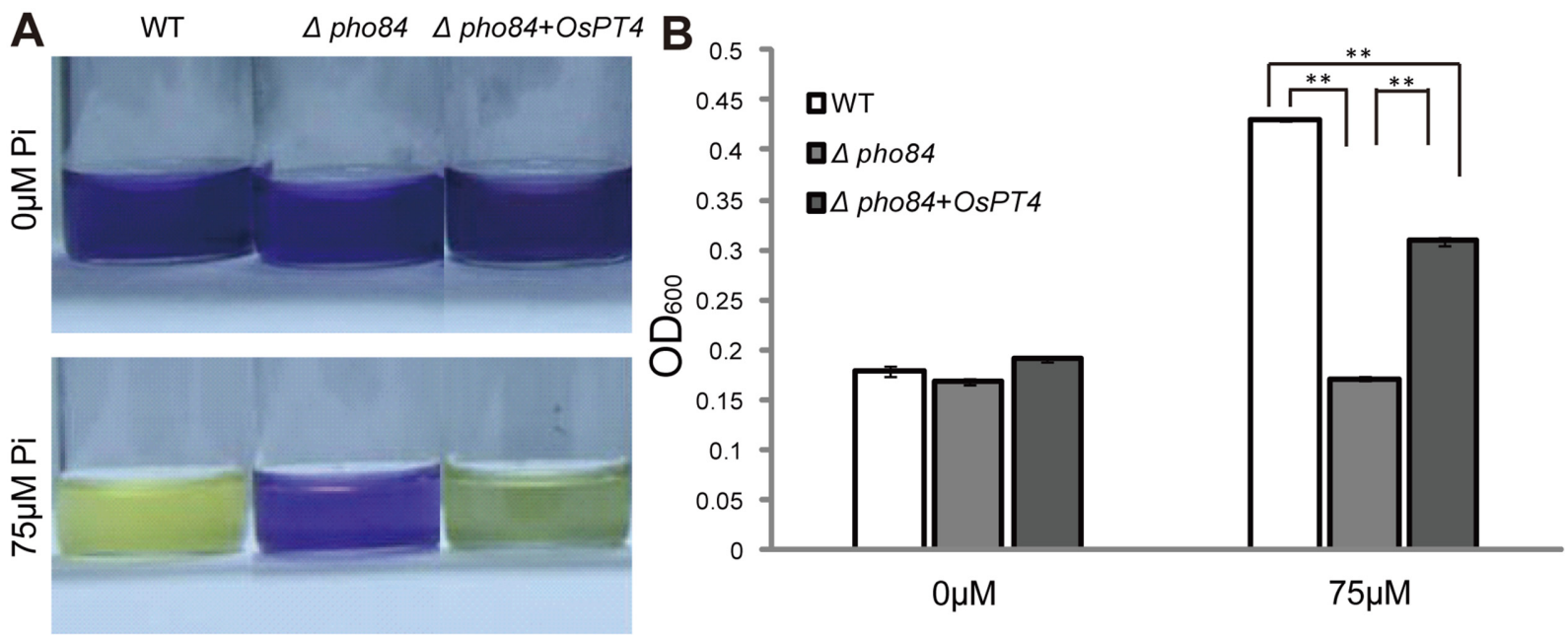

Fig 4. Function expression of OsPT4 in yeast. The yeast cells were grown in solution that had been stained for acidification. The $\mathrm{pH}$ indicator, bromocresol purple, did not change from blue to yellow until the yeast cells had significant growth in culture. The medium contained $0 \mu \mathrm{M}$ and $75 \mu \mathrm{M} \mathrm{Pi}$, respectively. A: Color shifts in wild-type (WT), yeast strain pho84 (control), and pho84+OsPT4, which express OsPT4 in pho84 grown on synthetic defined (SD) Ura mediums. B: Growth conditions of wild-type, pho84, and pho84 transformed with pYES2-OsPT4 generated in a 24-hour liquid culture under 0 $\mu \mathrm{M}$ and $75 \mu \mathrm{M}$ Pi conditions. Statistically significant differences are indicated: ${ }^{*} p<0.01$, Student's one-way ANOVA analysis.

doi:10.1371/journal.pone.0126186.g004

further study. Under sufficient Pi conditions, the transcript levels of OsPT4 in the two OsPT4Oe lines were 4.1-times and 14-times higher than those of wild-type, respectively, whereas the transcript abundance of OsPT4 decreased by 70\% in OsPT4-Ri plants (Fig 5A).

The transgenic plants were initially grown for 1 week in complete culture and then shifted to culture with HP, LP and normal Pi for 21 days. Using different Pi treatments, the phenotypes of OsPT4-Oe plants, including plant height, root length, tiller number, and the number of main roots, showed no obvious difference compared with wild-type (Fig 5E-5H). No significant differences were found in plant height and root length between OsPT4-Ri lines and wildtype plants (Fig 5C). However, the number of main roots and tiller numbers were decreased in OsPT4-Ri lines (Fig 5G and 5H).

Pi concentrations were measured in shoots and roots of the 21-day-old transgenic plants grown with different $\mathrm{Pi}$ levels. When grown without $\mathrm{Pi}$, all the lines possessed extremely low $\mathrm{Pi}$ concentrations both in roots and shoots and no significant difference in Pi concentrations were found among all the lines (Fig 5I and 5J). However, transgenic plants with overexpression of OsPT4 showed a increased Pi accumulation in roots when grown in normal and HP conditions. Interestingly, higher Pi seemed to cause a more significant increase of Pi in OsPT4-Oe plants in comparison with wild-type: the Pi concentration of OsPT4-Oe lines increased 1.6-times and 1.3-times, respectively, with $0.3 \mathrm{mM} \mathrm{Pi}$, and increased 1.73-times and 2.1-times with $1.5 \mathrm{mM}$ Pi. However, no significant difference was found in shoots of transgenic and wild-type plants grown with these two Pi concentrations. Additionally, the Pi concentrations in OsPT4-Ri plants were similar to those of wild-type under different Pi conditions.

\section{Alteration of Pi Concentration in OsPT4 Transgenic Plants Grown in Field}

The fact that the Pi concentration of OsPT4-Oe plants accumulated in roots suggested that OsPT4 may play an important role in rice Pi uptake. To understand the function of OsPT4 in Pi uptake, we measured the Pi concentration of wild type and OsPT4 transgenic plants grown in field with normal P concentration (available Pi of $14 \mathrm{mg} / \mathrm{kg}$ soil). The Pi concentration of 

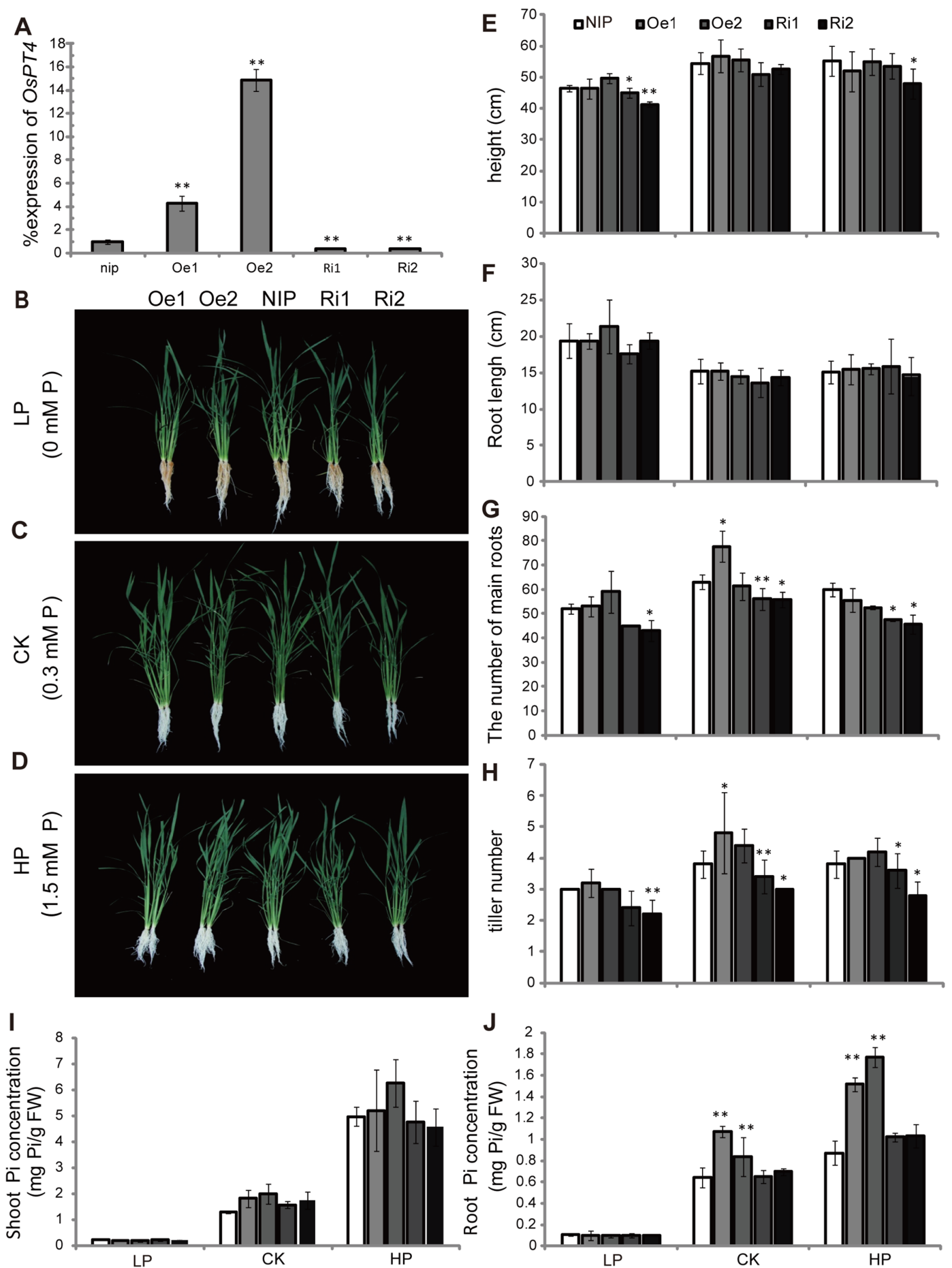

Fig 5. Characterization of wild-type and transgenic plants. A: Expression of OsPT4 in transgenic plants. Seven-day-old seedlings were transferred to nutrient solution for 2 weeks. RNA was extracted from the shoots of the seedling. Oe1, Oe2, Ri1, and Ri2 represented independent OsPT4-overexpressing and RNA interference lines. Relative expression levels are shown as percentages compared with wild-type shown as $100 \%$ expression. B, C, and D: Growth of wild-type and transgenic plants. Plants were grown in nutrient solutions to which $0 \mathrm{mM} \mathrm{Pi}(\mathrm{LP}), 1.5 \mathrm{mM} \mathrm{Pi} \mathrm{(HP),} \mathrm{and} 0.3 \mathrm{mM} \mathrm{Pi}(\mathrm{CK})$ were added for 21 days. E-H: Phenotypic analysis of OsPT4 transgenic plants. Height, root length, number of main roots, and tiller numbers were obtained from the 21 -dayold wild-type and transgenic plants grown in nutrient solutions with different $\mathrm{Pi}$ concentrations. Five plants per line were measured. I and $\mathrm{J}$ : The $\mathrm{Pi}$ concentration of shoots and roots in wild-type and transgenic plants. Data are means \pm SD of five biological replicates. Values are significantly different from those of wild-type: ${ }^{*} \mathrm{P}<0.05$ and ${ }^{*} \mathrm{P}<0.01$. (one-way ANOVA).

doi:10.1371/journal.pone.0126186.g005 


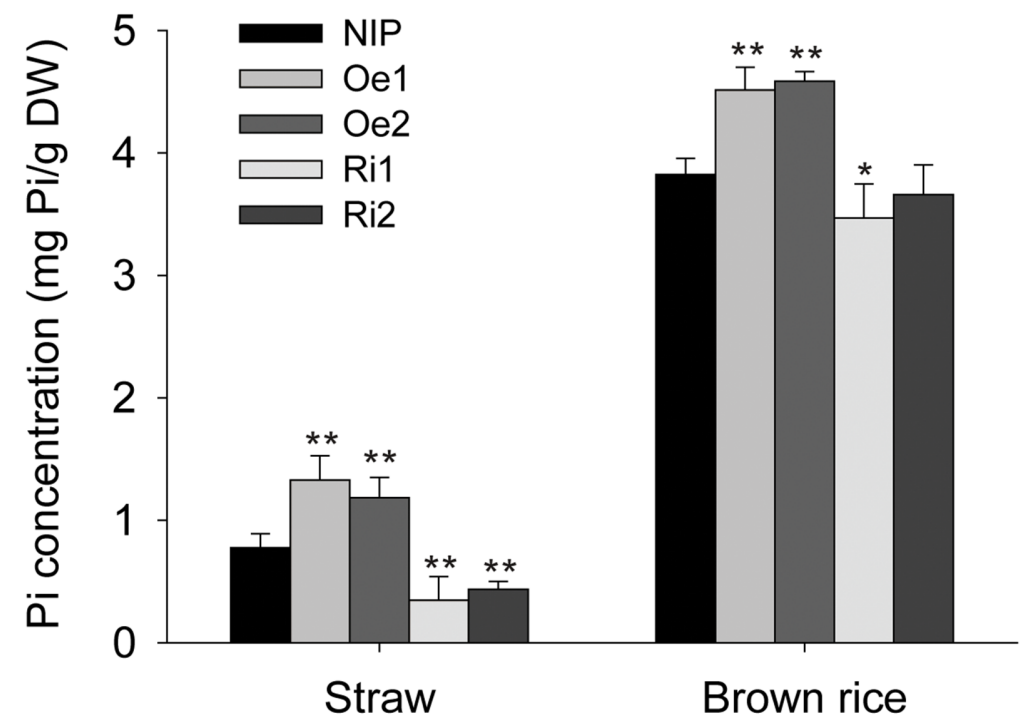

Fig 6. Pi concentration of the wild-type and transgenic plants in field. The rice including wild type and transgenic plants were grown in field. When plants grown to maturity, the phosphate concentration of straws and brown rice were measured. Data are means \pm SD of five biological replicates. Values are significantly different from those of wild-type: ${ }^{*} P<0.05$ and ${ }^{*} P<0.01$. (one-way ANOVA).

doi:10.1371/journal.pone.0126186.g006

transgenic plants in both straw and brown rice changed (Fig 6). The Pi concentration doubled in straw of the OsPT4-Oe plants compared with the wild type plants, whereas the straw Pi concentration in OsPT4-Ri decreased to about half of that in wild type plants. Furthermore, the Pi concentration of brown rice in OsPT4-Oe plants increased 26\% compared with wild type plants, whereas the Pi concentrations in OsPT4-Ri plants were slightly decreased. The biomasses of wild type and OsPT4 transgenic plants had no significant difference (S3 Fig).

\section{Altered Expression of Pi-signaling Genes in OsPT4 Transgenic Plants}

It was revealed that many important central regulators affecting Pi absorption and homeostasis had been functionally characterized in the pathways for Pi signaling, such as $\mathrm{OsPHO}$, OsPHR2, and OsSPX1. Therefore, we tested the expression of these three genes in transgenic plants and found that their expressions were changed in some way. The transcript level of $\mathrm{OsPHO} 2$ was increased in roots of OsPT4-Oe lines and dramatically decreased in leaves of OsPT4-Ri plants (Fig 7A). Additionally, OsPHR2 showed down-regulated expression in OsPT4-Ri lines, and the expression of OsSPX1 decreased slightly only in the roots of OsPT4-Ri plants (Fig 7B and 7C).

\section{Discussion}

\section{OsPT4 Is a Pi Transporter Involved in Pi Uptake}

Pht 1 family genes are present in nearly every living organism and are well-known to be related to Pi transport. In rice plants, there are approximately 13 members belonging to the Pht 1 family, and these genes are expected to be well-organized with a clear division of labor in Pi uptake and translocation [9]. A few members of the rice Pht 1 family have shown the ability to transport $\mathrm{Pi}$, and their exact roles in rice have also been clarified; OsPT1, OsPT2, OsPT6, and OsPT8 are all able to transport Pi and function in Pi uptake and translocation $[26,28,29]$. However, the roles of many other members of the rice Pht 1 family are still unclear. In the present study 

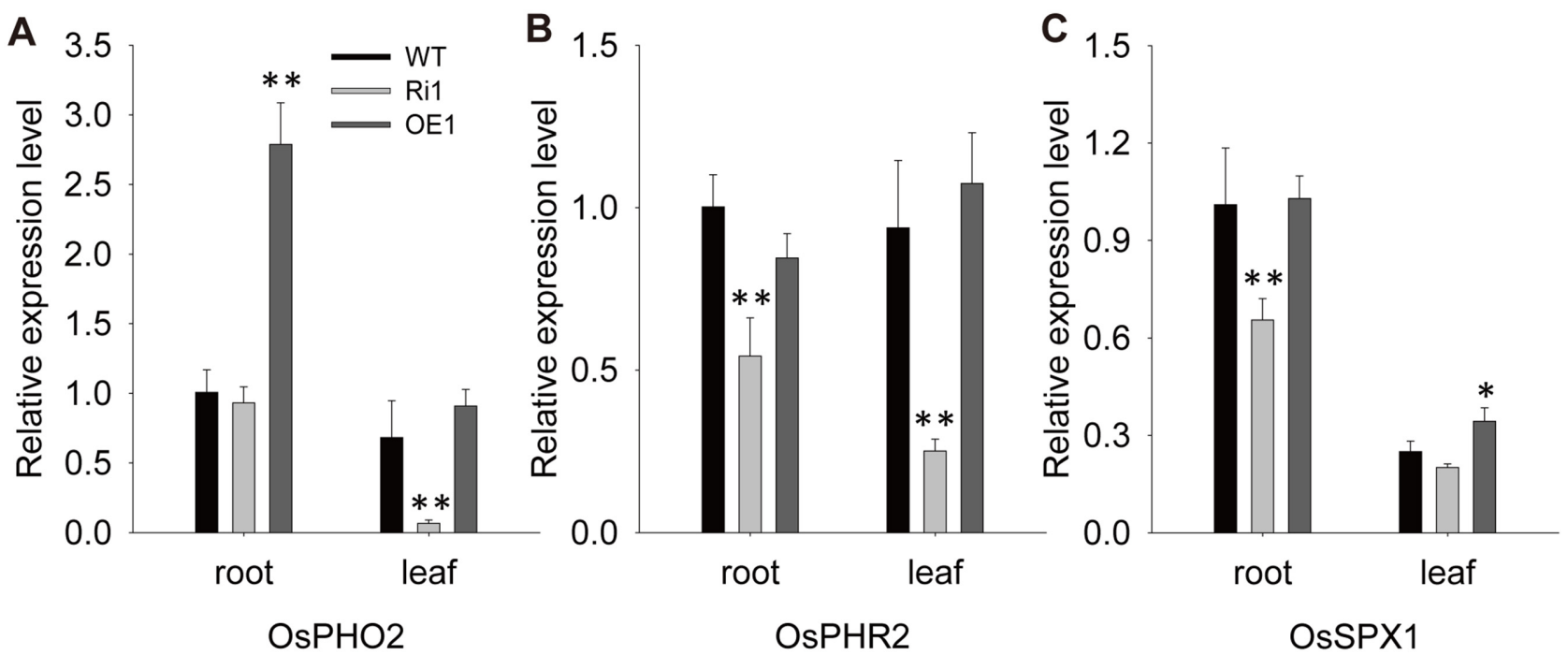

Fig 7. Expression of genes in wild-type transgenic plants under normal Pi condition. Expression of central transporters affecting Pi absorption and homeostasis in WT and transgenic plants. Error bars indicate $\pm S D(n=3) .\left({ }^{*} p<0.01\right.$ and ${ }^{*} P<0.05$, one-way ANOVA).

doi:10.1371/journal.pone.0126186.g007

we identified OsPT4 (another rice Pht1 family gene), which has a functional role in Pi transport. OsPT4 was shown here to be a functional Pi transporter and to contribute to Pi absorption. This conclusion is based on the following findings: (1) OsPT4 is an influx Pi transporter, at least in yeast, based on the fact that $O s P T 4$ can rescue yeast growth when expressed in yeast mutant pho84 (Fig 4); (2) OsPT4 encodes a plasma membrane protein, based on the GFP fluorescence from OsPT4-GFP fusion protein transformed into rice protoplasts (Fig 3); (3) OsPT4 is highly expressed in roots and specific to exodermis cells, based on the GUS staining analysis (Fig 2); (4) overexpression of OsPT4 in rice plants significantly increases root Pi concentration at different Pi levels compared with wild-type plants (Fig 5J) and (5) the Pi concentration of OsPT4 transgenic plants strictly respond to the OsPT4 transcripts change under field condition, overexpression of OsPT4 result in higher phosphate accumulation and suppression of OsPT4 decrease the total Pi amounts (Fig 6).

\section{Possible Role of OsPT4 in Pi Translocation and Homeostasis}

$\mathrm{Pi}$ is one of the most important elements for rice. In general, $\mathrm{Pi}$ is taken-up by rice roots, loaded into xylem, translocated to the shoots through root pressure and leaf transpiration, and, finally, distributed into various organs [1]. These processes always require multiple Pi transporters to collaborate with each other. During uptake, at least four Pi transporters other than OsPT4, including OsPT1, OsPT6, OsPT9, and OsPT10, have been shown to contribute to absorbing Pi at different Pi levels $[27,28,41]$. However, OsPT4 was the only transporter that was specific to exodermis cells in rice roots under normal Pi conditions, thus revealing that the role of OsPT4 is different from that of many other Pht1 family genes (S1 and S2 Figs). Rice roots have two types of sclerenchymatous cells, one at the endodermis (known as Casparian strip) and another at the exodermis. The latter in rice always acts as the first apoplastic barrier to avoid outer substance influx; therefore, the exodermis layer plays a key role in managing radial uptake of various mineral elements from the external solution, including $\mathrm{Pi}[42]$. Thus, we concluded that OsPT4 is probably constitutively expressed in the exodermis layer of rice roots and plays an important role in Pi uptake at different Pi levels (Fig 2E and 2F). Interestingly, OsPT4 was expressed in cells of root cortex under long period Pi deficiency. Since root cortex is involved in both apoplast and symplast pathways, the 
induced expression of OsPT4 in cortex would probably enhance the symplastic transport of Pi and consequently help rice plants to obtain more $\mathrm{Pi}$ at low Pi condition.

OsPT4 also shows high expression levels in various organs of rice shoots, such as leaves, ligules, nodes, internodes, and panicles, and its expression level in shoots is significantly induced by Pi-free conditions (Fig 2). This suggests that OsPT4 is possibly involved in Pi transport and translocation in these organs. Moreover, it is worth noting that OsPT4 showed the highest (even much higher than that in roots) expression level in flag leaves. Flag leaf is an essential tissue for the growth of rice panicles, and it plays a key role in remobilization of many mineral elements, from leaves to developing grains [43]. Until now, no Pi transporter had been shown to be localized in flag leaf and responsive to the remobilization of Pi from flag leaf to panicles. In our work, overexpressing OsPT4 resulted in much higher Pi concentrations in brown rice (Fig 6), although this could be a cumulative effect of increased root uptake, increased root-toshoot translocation and enhanced mobilization of Pi from other tissues to panicles. Thus, we deduced that OsPT4 was also probably involved in Pi remobilization from flag leaf to panicles. However, the exact mechanism will require further study.

Thorough reviews have shown that several Pi transporter genes are regulated by a few central regulators, such as OsPHR1 and OsPHR2, OsPHO1 and OsPHO2, and four SPX genes [44-46]. However, the expression of these regulator genes can also be affected by feedback from transcriptional changes in $\mathrm{PT}$ genes. In OsPT4 transgenic plants, we detected transcripts of OsPHR2, OsPHO2, and OsSPX1 and found that the expression of all three genes was changed somewhat (Fig 7). In shoots of plants overexpressing OsPT4, OsPHR2 and OsPHO2 expression levels were greatly down-regulated, consistent with the conclusion that OsPT4 is probably involved in shoot Pi translocation. In roots, OsPHR2 and OsSPX1 are down-regulated in OsPT4-RNAi plants, whereas the expression of OsPHR2 is up-regulated in OsPT4-

overexpressed plants. The changes in expression levels of these regulator genes might be a possible reason why many other Pi transporter genes are transcriptionally affected in OsPT4 transgenic plants.

\section{Supporting Information}

S1 Fig. Expression pattern in response to Pi availability. Detection of the expression alternation of OsPT4 in wild-type plants roots and shoots by real-time PCR analysis. Plants were grown to five-leaf stage in full nutrient solution, then transplanted into the solution without $\mathrm{Pi}$ one week and re-supplied Pi one day. Total RNA were extracted from plant tissues which were sampled at different time. Error bars indicate $\pm \mathrm{SD}(\mathrm{n}=3) .\left({ }^{* *} p<0.01\right)$.

S2 Fig. Tissue localization of OsPT4 in different Pi conditions. GUS staining of transgenic plants harboring the OsPT4 promoter. GUS fusion was observed. Expression of OsPT4 was shown in different tissues of rice supplied with $\mathrm{Pi}(\mathrm{A}-\mathrm{C})$ and without $\mathrm{Pi}(\mathrm{D}-\mathrm{F})$ for 21 days. A and D: leaf blade. $B$ and E: Transverse section of root maturation zone. $C$ and F: Enlarged images of $(\mathrm{H})$ and $(\mathrm{K})$.

(TIF)

S3 Fig. Biomass of WT and OsPT4 transgenic plants in the field. The rice including wild type and transgenic plants were grown in field. When plants grown to maturity, the biomass of wild type and OsPT4 transgenic plants were measured. Data are means \pm SD of five biological replicates. Values are significantly different from those of wild-type: ${ }^{*} \mathrm{P}<0.05$ and ${ }^{* *} \mathrm{P}<0.01$. (one-way ANOVA).

(TIF) 


\section{Acknowledgments}

We thank Professor Jian Xu for kindly providing the pM999-GFP vector.

\section{Author Contributions}

Conceived and designed the experiments: XL YY. Performed the experiments: YY JY LZ XC KL. Analyzed the data: YY JY. Contributed reagents/materials/analysis tools: XL. Wrote the paper: YY XL MY.

\section{References}

1. Raghothama KG, Karthikeyan AS. Phosphate acquisition. Plant and Soil. 2005; 274: 37-49.

2. Misson J, Raghothama KG, Jain A, Jouhet J, Block MA, Bligny R, et al. A genome-wide transcriptional analysis using Arabidopsis thaliana Affymetrix gene chips determined plant responses to phosphate deprivation. Proceedings of the National Academy of Sciences of the United States of America. 2005; 102: 11934-11939. PMID: 16085708

3. Vance $\mathrm{CP}$, Uhde-Stone $\mathrm{C}$, Allan DL. Phosphorus acquisition and use: critical adaptations by plants for securing a nonrenewable resource. New Phytologist. 2003; 157: 423-447.

4. Jouhet J, Marechal E, Block MA. Glycerolipid transfer for the building of membranes in plant cells. Progress in Lipid Research. 2007; 46: 37-55. PMID: 16970991

5. Weber MJ, Edlin G. Phosphate transport, nucleotide pools, and ribonucleic acid synthesis in growing and in density-inhibited 3T3 cells. J Biol Chem. 1971; 246: 1828-1833. PMID: 5102151

6. Chrispeels MJ, Crawford NM, Schroeder JI. Proteins for transport of water and mineral nutrients across the membranes of plant cells. Plant Cell. 1999; 11: 661-676. PMID: 10213785

7. Schachtman DP, Reid RJ, Ayling SM. Phosphorus Uptake by Plants: From Soil to Cell. Plant Physiol. 1998; 116: 447-453. PMID: 9490752

8. Doerner $\mathrm{P}$. Phosphate starvation signaling: a threesome controls systemic $\mathrm{P}(\mathrm{i})$ homeostasis. Current Opinion in Plant Biology. 2008; 11: 536-540. doi: 10.1016/j.pbi.2008.05.006 PMID: 18614391

9. Liu F, Chang XJ, Ye Y, Xie WB, Wu P, Lian XM. Comprehensive sequence and whole-life-cycle expression profile analysis of the phosphate transporter gene family in rice. Mol Plant. 2011; 4: 1105-1122. doi: 10.1093/mp/ssr058 PMID: 21832284

10. Rausch C, Bucher M. Molecular mechanisms of phosphate transport in plants. Planta. 2002; 216: 23-37. PMID: 12430011

11. Karthikeyan AS, Varadarajan DK, Mukatira UT, D'Urzo MP, Damsz B, Raghothama KG. Regulated expression of Arabidopsis phosphate transporters. Plant Physiology. 2002; 130: 221-233. PMID: 12226502

12. Mudge SR, Rae AL, Diatloff E, Smith FW. Expression analysis suggests novel roles for members of the Pht1 family of phosphate transporters in Arabidopsis. Plant Journal. 2002; 31: 341-353. PMID: 12164813

13. Shin H, Shin HS, Dewbre GR, Harrison MJ. Phosphate transport in Arabidopsis: Pht $1 ; 1$ and Pht $1 ; 4$ play a major role in phosphate acquisition from both low- and high-phosphate environments. Plant Journal. 2004; 39: 629-642. PMID: 15272879

14. Remy E, Cabrito TR, Batista RA, Teixeira MC, Sa-Correia I, Duque P. The Pht 1;9 and Pht1;8 transporters mediate inorganic phosphate acquisition by the Arabidopsis thaliana root during phosphorus starvation. New Phytologist. 2012; 195: 356-371. doi: 10.1111/j.1469-8137.2012.04167.x PMID: 22578268

15. Nagarajan VK, Jain A, Poling MD, Lewis AJ, Raghothama KG, Smith AP. Arabidopsis Pht 1;5 Mobilizes Phosphate between Source and Sink Organs and Influences the Interaction between Phosphate Homeostasis and Ethylene Signaling. Plant Physiology. 2011; 156: 1149-1163. doi: 10.1104/pp.111. 174805 PMID: 21628630

16. Versaw WK. A phosphate-repressible, high-affinity phosphate permease is encoded by the pho-5+ gene of Neurospora crassa. Gene. 1995; 153: 135-139. PMID: 7883177

17. Harrison MJ, van Buuren ML. A phosphate transporter from the mycorrhizal fungus Glomus versiforme. Nature. 1995; 378: 626-629. PMID: 8524398

18. Leggewie G, Willmitzer L, Riesmeier JW. Two cDNAs from potato are able to complement a phosphate uptake-deficient yeast mutant: identification of phosphate transporters from higher plants. Plant Cell. 1997; 9: 381-392. PMID: 9090882 
19. Daram P, Brunner S, Persson BL, Amrhein N, Bucher M. Functional analysis and cell-specific expression of a phosphate transporter from tomato. Planta. 1998; 206: 225-233. PMID: 9737001

20. Liu C, Muchhal US, Uthappa M, Kononowicz AK, Raghothama KG. Tomato phosphate transporter genes are differentially regulated in plant tissues by phosphorus. Plant Physiology. 1998; 116: 91-99. PMID: 9449838

21. Liu H, Trieu AT, Blaylock LA, Harrison MJ. Cloning and characterization of two phosphate transporters from Medicago truncatula roots: regulation in response to phosphate and to colonization by arbuscular mycorrhizal (AM) fungi. Mol Plant Microbe Interact. 1998; 11: 14-22. PMID: 9425684

22. Preuss $C P$, Huang $C Y$, Gilliham $M$, Tyerman SD. Channel-like characteristics of the low-affinity barley phosphate transporter PHT1;6 when expressed in Xenopus oocytes. Plant Physiol. 2010; 152: 1431-1441. doi: 10.1104/pp.109.152009 PMID: 20053709

23. Preuss $\mathrm{CP}$, Huang $\mathrm{CY}$, Tyerman SD. Proton-coupled high-affinity phosphate transport revealed from heterologous characterization in Xenopus of barley-root plasma membrane transporter, HvPHT1;1. Plant Cell and Environment. 2011; 34: 681-689. doi: 10.1111/j.1365-3040.2010.02272.x PMID: 21309796

24. Schunmann PHD, Richardson AE, Vickers CE, Delhaize E. Promoter analysis of the barley Pht $1 ; 1$ phosphate transporter gene identifies regions controlling root expression and responsiveness to phosphate deprivation. Plant Physiology. 2004; 136: 4205-4214. PMID: 15542491

25. Goff SA. A draft sequence of the rice genome (Oryza sativa L. ssp. japonica) Science. 2005; 309: 879-879.

26. Sun SB, Gu MA, Cao Y, Huang XP, Zhang X, Ai PH, et al. A Constitutive Expressed Phosphate Transporter, OsPht1;1, Modulates Phosphate Uptake and Translocation in Phosphate-Replete Rice. Plant Physiology. 2012; 159: 1571-1581. doi: 10.1104/pp.112.196345 PMID: 22649273

27. Wang XF, Wang YF, Pineros MA, Wang ZY, Wang WX, Li CY, et al. Phosphate transporters OsPHT1;9 and OsPHT1;10 are involved in phosphate uptake in rice. Plant Cell and Environment. 2014; 37: 1159-1170. doi: 10.1111/pce.12224 PMID: 24344809

28. Ai PH, Sun SB, Zhao JN, Fan XR, Xin WJ, Guo Q, et al. Two rice phosphate transporters, OsPht1;2 and OsPht1;6, have different functions and kinetic properties in uptake and translocation. Plant Journal. 2009; 57: 798-809. doi: 10.1111/j.1365-313X.2008.03726.x PMID: 18980647

29. Jia HF, Ren HY, Gu M, Zhao JN, Sun SB, Zhang X, et al. The Phosphate Transporter Gene OsPht1;8 Is Involved in Phosphate Homeostasis in Rice. Plant Physiology. 2011; 156: 1164-1175. doi: 10.1104/ pp.111.175240 PMID: 21502185

30. Zhang LH, Hu B, Li W, Che RH, Deng K, Li H, et al. OsPT2, a phosphate transporter, is involved in the active uptake of selenite in rice. New Phytologist. 2014; 201: 1183-1191. doi: 10.1111/nph.12596 PMID: 24491113

31. Paszkowski U, Kroken S, Roux C, Briggs SP. Rice phosphate transporters include an evolutionarily divergent gene specifically activated in arbuscular mycorrhizal symbiosis. Proceedings of the National Academy of Sciences of the United States of America. 2002; 99: 13324-13329. PMID: 12271140

32. Wu C, Li X, Yuan W, Chen G, Kilian A, Li J, et al. Development of enhancer trap lines for functional analysis of the rice genome. Plant Journal. 2003; 35: 418-427. PMID: 12887592

33. Yang M, Zhang Y, Zhang L, Hu J, Zhang X, Lu K, et al. OsNRAMP5 contributes to manganese translocation and distribution in rice shoots. J Exp Bot. 2014; 65: 4849-4861. doi: 10.1093/jxb/eru259 PMID: 24963001

34. Wang L, Xie WB, Chen Y, Tang WJ, Yang JY, Ye RJ, et al. A dynamic gene expression atlas covering the entire life cycle of rice. Plant Journal. 2010; 61: 752-766. doi: 10.1111/j.1365-313X.2009.04100.X PMID: 20003165

35. Hiei Y, Ohta S, Komari T, Kumashiro T. Efficient transformation of rice (Oryza sativa L.) mediated by Agrobacterium and sequence analysis of the boundaries of the T-DNA. Plant J. 1994; 6: 271-282. PMID: 7920717

36. Jefferson RA, Kavanagh TA, Bevan MW. GUS fusions: beta-glucuronidase as a sensitive and versatile gene fusion marker in higher plants. EMBO J. 1987; 6: 3901-3907. PMID: 3327686

37. Miao YS, Jiang LW. Transient expression of fluorescent fusion proteins in protoplasts of suspension cultured cells. Nature Protocols. 2007; 2: 2348-2353. PMID: 17947977

38. Basheer S, Samyn D, Hedstrom M, Thakur MS, Persson BL, Mattiasson B. A membrane protein based biosensor: Use of a phosphate- $-\mathrm{H}+$ symporter membrane protein (Pho84) in the sensing of phosphate ions. Biosensors \& Bioelectronics. 2011; 27: 58-63.

39. Zhou J, Jiao FC, Wu ZC, Li YY, Wang XM, He XW, et al. OsPHR2 is involved in phosphate-starvation signaling and excessive phosphate accumulation in shoots of plants. Plant Physiology. 2008; 146: 1673-1686. doi: 10.1104/pp.107.111443 PMID: 18263782 
40. Chen AQ, Hu J, Sun SB, Xu GH. Conservation and divergence of both phosphate- and mycorrhizaregulated physiological responses and expression patterns of phosphate transporters in solanaceous species. New Phytologist. 2007; 173: 817-831. PMID: 17286830

41. Seo HM, Jung Y, Song S, Kim Y, Kwon T, Kim DH, et al. Increased expression of OsPT1, a high-affinity phosphate transporter, enhances phosphate acquisition in rice. Biotechnology Letters. 2008; 30: 1833-1838. doi: 10.1007/s10529-008-9757-7 PMID: 18563580

42. Ishiyama K, Hayakawa T, Yamaya T. Expression of NADH-dependent glutamate synthase protein in the epidermis and exodermis of rice roots in response to the supply of ammonium ions. Planta. 1998; 204: 288-294. PMID: 9530872

43. Kumagai E, Hamaoka N, Araki T, Ueno O. Dorsoventral asymmetry of photosynthesis and photoinhibition in flag leaves of two rice cultivars that differ in nitrogen response and leaf angle. Physiol Plant. 2014; 151: 533-543. doi: 10.1111/ppl.12145 PMID: 24471973

44. Bari R, Datt Pant B, Stitt M, Scheible WR. PHO2, microRNA399, and PHR1 define a phosphatesignaling pathway in plants. Plant Physiol. 2006; 141: 988-999. PMID: 16679424

45. Liu F, Wang Z, Ren H, Shen C, Li Y, Ling HQ, et al. OsSPX1 suppresses the function of OsPHR2 in the regulation of expression of OsPT2 and phosphate homeostasis in shoots of rice. Plant J. 2010; 62: 508-517. doi: 10.1111/j.1365-313X.2010.04170.x PMID: 20149131

46. Cao Y, Yan Y, Zhang F, Wang HD, Gu M, Wu XN, et al. Fine characterization of OsPHO2 knockout mutants reveals its key role in Pi utilization in rice. J Plant Physiol. 2014; 171: 340-348. doi: 10.1016/j. jplph.2013.07.010 PMID: 24268791 